\title{
CARACTERIZAÇÃO DOS PACIENTES SUBMETIDOS A ESTOMAS INTESTINAIS EM UM HOSPITAL PÚBLICO DE TERESINA-PI
}

\author{
Maria Helena Barros Araújo Luz ${ }^{1}$, Danilo de Sousa Andrade², Hellen de Oliveira Amaral', Sandra Marina \\ Gonçalves Bezerra ${ }^{4}$, Cláudia Daniella Avelino Vasconcelos Benício ${ }^{5}$, Adinaide Cristina Almoedes Leal ${ }^{6}$
}

\footnotetext{
${ }^{1}$ Doutora em Enfermagem. Professor Adjunto da Universidade Federal do Piauí (UFPI), Orientadora do trabalho. Piauí, Brasil. E-mail: mhelenal@yahoo.com.br

${ }^{2}$ Acadêmico de Enfermagem da UFPI do $9^{\circ}$ período. Piauí, Brasil. E-mail: danilocanisso@hotmail.com

${ }^{3}$ Acadêmica de Enfermagem da UFPI do $9^{\circ}$ período. Piauí, Brasil. E-mail: hellenzinhamaral@yahoo.com.br

${ }^{4}$ Mestranda em Enfermagem da UFPI. Professora da Faculdade de Saúde, Ciências Humanas e Tecnológicas do Piauí e Universidade Estadual do Maranhão-Caxias. Piauí, Brasil. E-mail: sandramarina20@hotmail.com

${ }^{5}$ Especialista em Administração Hospitalar e em Estomaterapia. Enfermeira do Centro de Avaliação e Tratamento Avançado de Doenças Urológicas. Professora Substituta da UFPI. Piauí, Brasil. E-mail: claudiabenicioi@oi.com.br

${ }^{6}$ Especialista em Saúde Pública e Saúde da Família. Enfermeira do Hospital Getúlio Vargas. Professora da Faculdade Integral Diferencial. Piauí, Brasil. E-mail: adinaideenf@hotmail.com
}

\begin{abstract}
RESUMO: Estudo descritivo com abordagem quantitativa, objetivando caracterizar clientela estomizada atendida em hospital público quanto ao perfil sócio-demográfico e especificidades da cirurgia e estoma. Foram entrevistados 19 pacientes submetidos à cirurgia para realização de estomias intestinais, de abril a maio de 2007. Foi realizada análise estatística descritiva das variáveis estudadas. Predominaram estomizados do sexo masculino (52,63\%), na faixa etária entre 18 e 28 anos (31,58\%), procedentes do interior do Estado $(57,9 \%)$, casados $(47,37 \%)$, com baixa escolaridade $(62,16 \%)$ e baixa renda $(52,63 \%)$. A maioria das internações foi de emergência ( $84,21 \%)$, por causas obstrutivas $(63,16 \%)$, seguidas por causas traumáticas, resultantes da violência $(31,58 \%)$. Predominaram as colostomias $(84,21 \%)$, temporárias $(68,42 \%)$, apresentando efluentes líquidos $(63,16 \%)$, utilizando bolsas inadequadas $(47,37 \%)$ e com presença de hiperemia na pele periestoma (26,32\%). Conclui-se que a condição de ser estomizado, é geralmente vinculada a situações de emergência, associadas a acidentes, sendo necessário medidas preventivas e cuidados especializados.
\end{abstract}

DESCRITORES: Ostomia. Enfermagem perioperatória. Cuidados de enfermagem.

\section{CHARACTERIZATION OF PATIENTS SUBMITED TO INTESTINAL STOMAS IN A PUBLIC HOSPITAL OF TERESINA-PI, BRAZIL}

\begin{abstract}
The objective of this descriptive study with a quantitative approach was to characterize the clientele with stoma assisted at a public hospital concerning their socio-demographic profile and specificities of respective surgeries and stoma. Nineteen patients who underwent surgery for intestinal stomas were interviewed during April and May of 2007. From the data tabulation and descriptive statistic analysis of distributions of simple frequency and percentiles of the variables studied, the following characteristics were verified among patients with stoma: male (52.63\%), in the age group between 18 and 28 years (31.58\%), coming from the interior of the State (57.9\%), married (47.37\%), low education level (62.16\%) and low income (52.63\%). Most admissions were emergencies (84.21\%), with others for obstructive causes $(63.16 \%)$, followed by traumatic causes, resulting from violence (31.58\%). Predominated the colostomies $(84.21 \%)$, temporary $(68.42 \%)$, presenting liquid tributaries $(63.16 \%)$, using inappropriate bags $(47.37 \%$ ) and with hyperemia presence in the peristoma skin (26.32\%). It is concluded that this clientele is usually associated to emergencies, associated to accidents, thats requires prevention and specialized care.

DESCRIPTORS: Ostomy. Perioperative nursing. Nursing care.
\end{abstract}

\section{CARACTERIZACIÓN DE LOS PACIENTES OSTOMIZADOS INTESTINALES EN UN HOSPITAL PÚBLICO DE TERESINA-PI}

RESUMEN: Estudio descriptivo con abordaje cuantitativo, cuyo objetivo fue caracterizar el paciente ostomizado atendido en un hospital público, en relación al perfil sociodemográfico y a las especificidades de la cirugía y del estoma. Fueron entrevistados 19 pacientes sometidos a cirugía para la realización de ostomías intestinales, en el período de abril a mayo de 2007. A través de la tabulación de los datos, con distribución de la frecuencia simple y porcentaje, se constató que predominaron ostomizados de sexo masculino (52,63\%), entre 18 e 28 años (31,58\%), procedentes del interior del Estado de Piauí (57,90\%), casados (47,37\%), con baja escolaridad (62,16\%) y baja renta $(52,63 \%)$. La mayoría de las internaciones fue de emergencia $(84,21 \%)$, por causas obstructivas $(63,16 \%)$, seguidas de causas traumáticas, resultantes de la violencia $(31,58 \%)$. Predominaron las colostomías $(84,21 \%)$, temporarias $(68,42 \%)$, presentando afluentes líquidos $(63,16 \%)$, utilizando bolsas inadecuadas $(43,37 \%)$ y con presencia de hiperemia en la piel periestoma (26,32\%). Se concluye que los pacientes ostomizados, por las situaciones de emergencia, asociadas a accidentes, presentan características que requieren de medidas preventivas y cuidados especializados.

DESCRIPTORES: Ostomía. Enfermería perioperatoria. Atención de enfermería. 


\section{INTRODUÇÃO}

Estoma é uma palavra derivada de dois termos gregos, os e tomia, que significam abertura de uma boca ou comunicação entre um órgão interno e o exterior, com a finalidade de suprir a função do órgão afetado, em diversos sistemas orgânicos. ${ }^{1}$

Os estomas intestinais podem ser realizados no segmento do intestino delgado ou grosso que é trazido à superfície abdominal através de uma incisão cirúrgica, para drenagem de fezes e gases. Suas características físicas quanto ao tipo, localização, tamanho, forma, superfície, contorno e protrusão podem variar de acordo com a técnica cirúrgica utilizada, o segmento exteriorizado, a causa básica e o tempo de permanência. ${ }^{2}$

As causas dos estomas intestinais de eliminação são numerosas, diversificadas e possuem várias formas de classificação: quanto ao segmento exteriorizado, ao tempo de permanência, à forma de exteriorização, à continência, à maturação e à via de acesso. ${ }^{3}$

O paciente que se submete a uma estomia necessita de cuidados específicos, um acompanhamento especializado que atenda às suas necessidades psicobiológicas, psicossociais e psicoespirituais, e requer um plano de cuidados de enfermagem abrangente e contínuo. ${ }^{2} \mathrm{Na}$ indicação de uma estomia, deve-se fazer um planejamento pré-operatório criterioso a fim de se evitar problemas pós-operatórios.

Uma boa assistência de enfermagem deve começar no pré-operatório, com a avaliação, orientações e cuidados com o preparo necessário para o enfrentamento da cirurgia. E deve ser continuado durante o período em que o paciente permanecer estomizado, o que pode ser permanentemente. O cliente deve ser muito bem orientado, ensinado e treinado quanto às habilidades para assumir o seu autocuidado, envolvendo todos os cuidados necessários que deve tomar em se tratando da manipulação do estoma, como: limpeza da pele periestomal, especificações e disponibilidade dos dispositivos..$^{2,4}$

É papel do enfermeiro fornecer apoio emocional, estar atento à integridade cutânea, ensinar o paciente a trocar corretamente os dispositivos, observar se as necessidades hídricas e nutricionais estão sendo supridas e ficar atento a sinais de complicações. $^{2}$

Nesse sentido, torna-se importante focar a problemática que envolve o sujeito estomizado na perspectiva do cuidar na área da enfermagem, destacando que algumas pesquisas já foram realizadas em Teresina abordando aspectos diversos dessa clientela como: a sexualidade da mulher estomizada, prevalência de complicações do estoma e pele periestomal, perfil sócio-demográfico e epidemiológico de pessoas estomizadas participantes de um Programa de Atendimento ao Estomizado, desenvolvido no ambulatório do Centro Integrado de Saúde Lineu Araújo e um outro em instituição hospitalar filantrópica, de referência no tratamento do câncer. ${ }^{5-6}$ Portanto, todos com características bastante diferenciadas de onde foi realizado o presente estudo. Por conseguinte, buscamos ampliar o conhecimento sobre essa clientela verificando as características que apresentam o sujeito atendido em um hospital público, de referência para todo o estado do Piauí e estados vizinhos, inclusive com serviço de urgência e emergência.

Dessa forma considera-se relevante a caracterização do perfil dessa clientela como subsídios sobre a dimensão dos sujeitos envolvidos nessa problemática, condições de sua ocorrência e fatores associados. Essas informações visam o melhor atendimento ao cliente, em todos os níveis de atenção à saúde, desde a atenção primária, passando pela secundária e alcançando a terciária, ampliando o papel e o compromisso profissional do enfermeiro desde a prevenção até o processo reabilitatório do cliente, imprimindo no cuidar a mediação da educação na busca da autonomia para o autocuidado, e na socialização o fortalecimento para a defesa dos seus direitos de cidadania, dignidade e qualidade de vida. ${ }^{7-9}$

A partir dessa perspectiva, este estudo tem como objetivo caracterizar a clientela estomizada atendida em um hospital público de Teresina-PI.

Este estudo torna-se relevante pela escassez de informações e publicações nessa área, cuja problemática é pouco conhecida e explorada, assim como a clientela precariamente, assistida, apesar de bastante freqüente na prática profissional. ${ }^{6}$

\section{METODOLOGIA}

Trata-se de um estudo exploratório e descritivo, com abordagem quantitativa realizado em um hospital público de Teresina-PI. Este estudo compreendeu uma amostra constituída por 19 pacientes que se encontravam internados no Serviço de Pronto-Socorro e na Clínica Cirúrgica, no período de 17 de abril a 17 de maio de 2007.

Os critérios para a seleção e inclusão dos sujeitos do estudo foram: ser portador de estomia 
intestinal de eliminação, em pós-operatório, estar consciente, lúcido e orientado, e concordar em participar da pesquisa.

O projeto de pesquisa foi previamente, submetido à apreciação do Comitê de Ética em Pesquisa da Universidade Federal do Piauí e do referido hospital, obtendo aprovação em parecer $\mathrm{N}^{\mathrm{o}}$ 0021-07 expedido em 30.04.07 e $\mathrm{N}^{\circ} 0499$ expedido em 3 de abril de 2007, respectivamente, tendo sido cumpridas as exigências da Resolução No 196/96, do Conselho Nacional de Saúde/Ministério da Saúde no decorrer de sua execução, tendo os sujeitos da pesquisa assinado o Termo de Consentimento Livre e Esclarecido.

A coleta de dados foi realizada mediante entrevista, utilizando um roteiro semi-estruturado, complementado com leitura do prontuário do cliente, visando preencher dados sobre a caracterização da clientela. Utilizou-se as seguintes variáveis: idade, sexo, estado civil, naturalidade, escolaridade, profissão, renda familiar, caráter da internação, antecedentes pessoais e familiares, causa da realização da estomia. Além disso, foram realizados observação e exame físico em relação à cirurgia e ao estoma, em que foi avaliada a localização, coloração, tipo de estomia, tipo de exteriorização da alça, características do efluente, condições da pele periestoma, tempo de permanência e tipo de dispositivo em uso.

Após a coleta, foi realizada análise descritiva dos dados (freqüência e porcentagem), que foram analisados, discutidos, correlacionados e apresentados em tabelas.

\section{RESULTADOS E DISCUSSÃO}

Os 19 sujeitos que compuseram a amostra foram caracterizados quanto a aspectos sócio-demográficos e específicos da cirurgia e do estoma.

Os resultados evidenciaram que a maioria dos portadores de estomia é do sexo masculino $(52,63 \%)$. O predomínio do sexo masculino coincide com achados similares em estudos anteriores, ${ }^{10}$ assim como, particularmente, nesse estudo o cenário envolveu o serviço de emergência e a causa básica encontra-se associada a trauma e violência em que o sexo masculino está mais exposto. Tais dados podem ser observados na Tabela 1.

Houve predominância de pacientes casados $(47,37 \%)$, seguidos por solteiros $(36,84 \%)$, viúvos $(10,53 \%)$ e pacientes com outros tipos de relação conjugal $(5,26 \%)$ (Tabela 1). Este resultado se assemelha a outros estudos. ${ }^{5} \mathrm{~A}$ análise desse dado faz-se importante porque a situação conjugal do estomizado, sua vida sexual, está diretamente relacionada aos problemas resultantes da estomia. A vida sexual dos estomizados sofre restrições, causadas em parte por mutilações anatômicas resultantes de ressecções envolvendo músculos e nervos relacionados ao funcionamento dos órgãos sexuais e, também, psico-emocional por sentimentos de vergonha e desinteresse sexual. ${ }^{6,11}$

Tabela 1 - Dados sócio-demográficos de pacientes submetidos à estomias intestinais. Teresina-PI, 2007

\begin{tabular}{lrr}
\hline Características & $\mathbf{n}$ & $\%$ \\
\hline Sexo & 10 & 52,63 \\
$\quad$ Masculino & 9 & 47,37 \\
$\quad$ Feminino & & \\
Faixa etária & 6 & 31,58 \\
$\quad 18 \mid-28$ & 1 & 5,26 \\
$28 \mid-38$ & 5 & 26,32 \\
$38 \mid-48$ & 2 & 10,53 \\
$48 \mid-58$ & 2 & 10,53 \\
$58 \mid-68$ & 2 & 10,53 \\
$68 \mid-78$ & 1 & 5,26 \\
$\geq 78$ & & \\
Estado civil & 7 & 36,84 \\
Solteiro & 9 & 47,37 \\
Casado & 2 & 10,53 \\
Viúvo & 1 & 5,26 \\
Outros & & \\
Escolaridade & 6 & 31,58 \\
Analfabeto & 6 & 31,58 \\
Ensino fundamental incompleto & 3 & 15,79 \\
Ensino fundamental completo & 3 & 15,79 \\
Ensino médio incompleto & 1 & 5,26 \\
$\quad$ Ensino médio completo & & \\
Renda Familiar & 5 & 26,32 \\
Até 1 salário mínimo & 52,63 \\
De 2 a 3 salários mínimos & 10 & 21,05 \\
$\quad$ Acima de 3 salários mínimos & 4 & \\
\hline & &
\end{tabular}

Quanto ao nível de escolaridade (Tabela 1), percebeu-se que a maioria dos indivíduos entrevistados enquadra-se nas categorias de analfabetos $(31,58 \%)$ e com ensino fundamental incompleto $(31,58 \%)$, somente $15,79 \%$ possuíam ensino fundamental completo, $15,79 \%$ ensino médio incompleto e $5,26 \%$ ensino médio completo. Na clientela estudada não houve nenhum caso com formação superior ou $3^{\circ}$ grau completo. É bom salientar que o estudo foi realizado em um hospital público de 
ensino e o perfil encontrado foram pessoas de baixa renda e escolaridade. O maior percentual de pacientes estomizados encontra-se no $1^{\circ}$ grau incompleto. O conhecimento da escolaridade dos estomizados pelo enfermeiro permite que este se adeque ao nível de entendimento do paciente., ${ }^{7,12}$

A maioria dos pacientes estomizados investigados possui renda familiar compreendida entre dois e três salários mínimos (52,63\%) (Tabela 1). O conhecimento desta variável faz-se importante porque torna evidente a dificuldade dos estomizados em adquirir os dispositivos necessários ao autocuidado. Nesse caso, cabe ao enfermeiro encaminhar os pacientes a grupos de apoio ou Associação de Estomizados, programas especiais oferecidos pelo Sistema Único de Saúde e inclusive distribuidores de equipamentos disponíveis no mercado. ${ }^{5-7}$

As profissões dos entrevistados também confirmam sua baixa escolaridade e renda: 47,37\% eram lavradores, $15,79 \%$ vendedores ambulantes, 10,53\% donas de casa, 10,53\% estudantes, 5,26\% lavadores de carro, 5,26\% cobradores de ônibus, e $5,26 \%$ professores. Com relação à naturalidade dos pacientes submetidos às estomias intestinais, pôde-se constatar que $57,9 \%$ provêm do interior do Piauí, enquanto que $21,05 \%$ são do estado do Maranhão, e 21,05\% são de Teresina. Esta grande demanda de pacientes do interior do Piauí e de estados vizinhos, principalmente o Maranhão, dá-se porque o hospital onde foi realizada a pesquisa é um centro de referência para tratamentos de saúde do Norte e Nordeste do país.

Ao investigar a faixa etária desta clientela, cujos dados podem ser observados na Tabela 1, constatou-se que $31,58 \%$ encontram-se na faixa etária de 18 a 28 anos, seguido, 26,32\% entre 38 e 48 anos de idade. Esta predominância de estomas entre jovens e adultos está associada a uma maior freqüência de doenças inflamatórias intestinais nessas faixas etárias tal como a doença de Crohn e retocolite ulcerativa, nas quais ocorrem manifestações inflamatórias e obstrutivas assim como também, as perfurações intestinais decorrentes de traumas por armas branca e de fogo conforme evidenciadas na Tabela $2 .{ }^{12}$

Com relação à internação, a maioria dos entrevistados $(84,21 \%)$ deu entrada no hospital em caráter de emergência, enquanto que apenas uma pequena parcela $(15,79 \%)$ realizou a cirurgia em caráter eletivo. O tipo de internação está diretamente relacionado com as causas que motivaram as estomias. Destacaram-se as causas clínicas obstrutivas com $57,89 \%$ e as traumáticas por armas brancas e armas de fogo, com $21,05 \%$ e $10,53 \%$ respectivamente. As motivadas por síndromes inflamatórias correspondem a 10,53\% do total de pacientes estomizados (Tabela 2).

Tabela 2 - Causas de cirurgias construtoras de estomias intestinais. Teresina-PI, 2007

\begin{tabular}{lcc}
\hline Causas & $\mathbf{n}$ & $\%$ \\
\hline Traumas & & \\
Por arma branca & 4 & 21,05 \\
Por arma de fogo & 2 & 10,53 \\
Clínicas & & \\
$\quad$ Obstrutiva & 11 & 57,89 \\
$\quad$ Inflamatória & 2 & 10,53 \\
\hline Total & $\mathbf{1 9}$ & $\mathbf{1 0 0 , 0 0}$ \\
\hline
\end{tabular}

As causas obstrutivas, geralmente determinam internações para cirurgias em caráter de emergência. Nesses casos, a dor abdominal foi uma manifestação clínica freqüente. As causas clínicas inflamatórias foram detectadas com menor frequiência, mas também sugerem internações de emergência por suas manifestações clínicas, resultantes de obstrução e perfuração. Às causas traumáticas quer por armas brancas ou armas de fogo, caracterizam o tipo de internação de emergência e refletem a realidade social e a presença da violência no nosso cotidiano. As estomias resultantes de causas traumáticas ocorreram, em sua maioria, em pacientes pertencentes a uma faixa etária mais jovem (18 - 38 anos). Isso pode ser explicado por estas pessoas estarem mais suscetíveis à violência, por se exporem mais. ${ }^{1}$ Este índice também evidencia que o hospital onde a pesquisa foi realizada é um centro de referência para o atendimento de emergências.

Entre os entrevistados, a maioria usava a bolsa coletora precária, menos recomendada $(47,36 \%)$ - bolsa transparente, não recortável, sem protetor de pele e sem adesivo hipoalergênico. Este tipo de bolsa embora possibilite boa visualização para observação das condições do estoma no período perioperatório, não possui boa aderência, necessitando ser trocada com freqüência, produzindo irritação e lesão da pele periestoma. Percebe-se a falta de bolsas adequadas disponíveis na instituição e de orientação aos pacientes quanto ao uso das bolsas no pós-operatório mediato e tardio. Os demais indivíduos estavam divididos igual- 
mente entre os dois grupos, que faziam uso de bolsas transparentes ou opacas, drenáveis e com protetor da pele periestoma e adesivo hipoalergênico (26,32\% cada). Esses são os tipos de bolsas coletoras mais adequadas conforme especificações da Sociedade Brasileira de Estomaterapia. ${ }^{13}$ Esses dados podem ser observados na Tabela 3.

Tabela 3 - Equipamentos utilizados para estomas intestinais. Teresina-PI, 2007

\begin{tabular}{lcc}
\hline Equipamentos & $\mathbf{n}$ & $\mathbf{\%}$ \\
\hline $\begin{array}{l}\text { Bolsa transparente não recortável, sem } \\
\text { adesivo hipoalergênico }\end{array}$ & 9 & 47,36 \\
$\begin{array}{l}\text { Bolsa drenável transparente com uma aber- } \\
\text { tura recortável, com adesivo hipoalergênico }\end{array}$ & 5 & 26,32 \\
$\begin{array}{l}\text { Bolsa drenável opaca com uma abertura } \\
\text { recortável, com adesivo hipoalergênico }\end{array}$ & 5 & 26,32 \\
\hline Total & 19 & $\mathbf{1 0 0 , 0 0}$ \\
\hline
\end{tabular}

Quanto à caracterização das estomias intestinais, $15,79 \%$ eram ileostomias (sendo $66,67 \%$ com abertura em alça, e 33,33\% com abertura em duas bocas), e $84,21 \%$ colostomias (Tabela 4 ), sendo que, destas, 50\% localizaram-se no colo descendente, $25 \%$ no colo ascendente, $18,75 \%$ no colo transverso e $6,25 \%$ no sigmóide.

Tabela 4 - Distribuição dos tipos de estomia realizadas. Teresina-PI, 2007

\begin{tabular}{lcc}
\hline Tipos estomias intestinais & $\mathbf{n}$ & $\boldsymbol{\%}$ \\
\hline lleostomias & 3 & 15,79 \\
Colostomias & & \\
\hline Total & 16 & 84,21 \\
\hline
\end{tabular}

Vale ressaltar que no grupo das colostomias, $68,75 \%$ possuíam abertura terminal, $18,75 \%$ em duas bocas e $12,5 \%$ em alça. As colostomias em alça, geralmente são realizadas como complemento de um outro procedimento cirúrgico, podendo ser indicadas em condições eletivas ou de urgência. ${ }^{4}$

Os tipos de efluentes observados perfaziam $63,16 \%$ para líquidos e $36,84 \%$ para pastoso. Não se encontrou nenhum caso de fezes formadas, embora a maioria das estomias fossem colostomias, devendo-se a maior freqüência de eliminações líquidas à proximidade da realização da cirurgia.
No caso das ileostomias, a eliminação é freqüente e aquosa no período pós-operatório imediato, e por isso o paciente perde muito líquido, podendo alcançar de 1000 - 1500 ml/ dia. Nesse caso, devese controlar cuidadosamente a entrada e saída de fluidos, para evitar qualquer alteração do equilíbrio hidroeletrolítico, causado por um excesso de eliminação produzida pelo estoma, uma vez que a evacuação de uma ileostomia não pode ser controlada voluntariamente. ${ }^{1}$

Com relação às colostomias, da mesma forma que nas ileostomias, não há o controle voluntário das eliminações, fezes e gases são drenados continuamente. Porém, em alguns casos, pode-se realizar o condicionamento intestinal através da irrigação das colostomias. ${ }^{6}$

Quanto às condições da pele periestoma, $68,42 \%$ dos indivíduos não apresentavam queimadura, em 26,32\% foi observado hiperemia e $5,26 \%$ cianose. Os achados referentes à hiperemia assemelham-se aos encontrados em vários estudos, em que um terço dos pacientes investigados apresentou dermatites periestoma. ${ }^{5} \mathrm{~A}$ hiperemia pode ser justificada pela inadequação dos equipamentos utilizados nas estomias, pois as bolsas, que necessitam de trocas freqüentes, proporcionam a retirada das camadas protetoras da pele, provocando hiperemia e erosões. ${ }^{5}$ A cianose observada pode ocorrer por deficiência de perfusão sanguínea do estoma, sendo esta geralmente relacionada com inadequada perfusão sanguínea relacionada às condições da alça intestinal e da técnica cirúrgica utilizada. ${ }^{4}$

O tempo de permanência da estomia intestinal variou em $68,42 \%$ para temporário e 31,58\% para permanente. Em estudos recentes houve divergência desses dados em que $81,25 \%$ dos entrevistados possuíam estoma definitivo e, 18,75\% possuíam estoma temporário; ${ }^{5}$ mas, por outro lado, são confirmados por outras pesquisas em que os estomas temporários perfizeram $60,98 \%$ do total, enquanto que $39,02 \%$ das estomias foram definitivas. ${ }^{14}$ As divergências nos resultados relacionados à permanência das estomias devem-se ao fato desta permanência estar diretamente relacionada com as causas que motivaram a construção dos estomas, quando a definitiva se associa mais freqüentemente ao câncer colorretal e urogenital e, a temporária aos traumas.

\section{CONCLUSÃO}

Este estudo possibilitou dimensionar e caracterizar os pacientes submetidos a estomas intestinais 
em um hospital de ensino em Teresina-PI, no período de abril a maio de 2007. Esses pacientes constituem uma realidade freqüente na prática profissional cotidiana do enfermeiro, que abrange uma ampla diversidade de cenários desde a atenção básica, os serviços ambulatoriais, unidades de internação hospitalar em especialidades, de menor ou maior complexidade, e até programas de reabilitação.

A caracterização destes pacientes nos permitiu as seguintes conclusões com relação aos aspectos sócio-demográficos: predominância de estomizados do sexo masculino; 18 e 28 anos; procedentes do interior do Estado; maior percentual de casados; de baixa escolaridade, analfabetos ou com ensino fundamental incompleto; com renda de um a três salários mínimos.

Com relação à realização das cirurgias e caracterização das estomias, concluímos que houve predomínio de internações em caráter de emergência; por causas obstrutivas, seguidas por causas traumáticas resultantes da violência; predominância de colostomizados; com estomas temporários; eliminação de efluentes líquidos; e presença de hiperemia na pele periestoma por uso de bolsas coletoras que não oferecem proteção adequada à pele de quase metade dos entrevistados.

Nesse sentido cabe ao enfermeiro atuar junto à equipe multiprofissional no cuidado direto no perioperatório e junto aos gestores para aquisição de bolsas coletoras com as devidas especificações e indicações para o cuidado adequado a cada tipo e características do estoma, favorecendo a observação, o manuseio, a adaptação do paciente e o treinamento para o autocuidado. Desse modo, pode-se reduzir o desconforto e complicações para o paciente, melhorando a qualidade do cuidado de enfermagem e dos serviços de saúde.

O número considerável de clientes submetidos às estomias intestinais decorrentes de causas traumáticas por armas de fogo e armas brancas reflete as condições sociais alarmantes em âmbito regional e nacional, onde a violência configura-se como um fator relevante e influenciador em todas as esferas da sociedade, moldando comportamentos, gerando despesas cada vez maiores aos cofres públicos e exacerbando ainda mais, a séria problemática da saúde pública em nosso país.

Além do mais, a assistência a essa clientela demanda insumos especiais apropriados e profissionais capacitados para lidar com os diversos problemas secundários (distúrbios físicos, emocionais, sociais), essenciais à recuperação e reabilitação desses pacientes.
Vale ressaltar ainda que a faixa etária mais acometida por essa problemática é justamente a adulta, economicamente ativa, tendo como causa principalmente, traumas e violência, implicando na redução da oferta de mão-de-obra, na geração de serviços e riquezas, ocasionando mais gastos ao Estado com a invalidez/aposentadoria precoce assim como de idosos, que constituem um segmento com acentuado crescimento populacional, geralmente por causas relacionadas ao câncer colorretal e urogenital.

Portanto, além dos inúmeros problemas intrínsecos que envolvem as causas básicas e os procedimentos técnicos-operacionais das cirurgias as quais resultam os estomas, que demandam cuidados hospitalares e pós-hospitalares especializados, existem os distúrbios de caráter psicossocial, físicos e emocionais que influenciam diretamente a reabilitação dos pacientes e dificultam o seu retorno ao convívio social, cabendo à Enfermagem dedicar-se de forma holística ao cuidado dessa clientela, cada vez mais freqüente no cotidiano da sua prática profissional.

\section{REFERÊNCIAS}

1. Breckman B. Enfermería del estoma. Madrid (ES): Interamericana, McGraw- Hill, 1987.

2. Brunner LS, Suddarth DS. Tratamento de pacientes com distúrbios intestinais e retais. In: Brunner LS, Tratado de enfermagem médico-cirúrgica. $10^{\mathrm{a}} \mathrm{ed}$. Rio de Janeiro (RJ): Guanabara Koogan, 2006. p. 1087-133.

3. Martins Júnior A, Rocha JJR. Tipos de estomas intestinais. In: Crema E, Silva R. Estomas: uma abordagem interdisciplinar. Uberaba (SP): Pinti, 1997. p. 41-64.

4. Crema E, Silva R. Estomas - uma abordagem interdisciplinar. Uberaba (SP): Pinti, 1997.

5. Macêdo MS, Nogueira LT, Luz, MHBA. Perfil dos estomizados atendidos em hospital de referência em Teresina. Rev Estima. 2005 Out-Dez; 3(4):25-8.

6. Luz MHBA. A dimensão cotidiana da pessoa estomizada: um estudo de enfermagem no referencial de Martin Heidegger [tese]. Rio de Janeiro (RJ): Universidade Federal do Rio de Janeiro. Escola de Enfermagem Anna Nery; 2001.

7. Bellato R, Pereira WR, Maruyama SAT, Oliveira PC A convergência cuidado-educação-politicidade: um desafio a ser enfrentado pelos profissionais na garantia aos direitos à saúde das pessoas portadoras de estomias. Texto Contexto Enferm. 2006 Abr-Jun; 15(2):334-42.

8. Santos VLCG. Cuidando do estomizado: análise da trajetória no ensino, pesquisa e extensão [tese]. São Paulo (SP): Universidade de São Paulo; 2006. 
9. Cascais AFMV, Martini JG, Almeida PJS O impacto da ostomia no processo de viver. Texto Contexto Enferm 2007 Jan-Mar; 16(1):163-7.

10. Von Bahten LCV, Nicoluzzi, JL.; Silveira, F.; Nicollelli GM; Kumagai, LY; Lima, VZ Morbimortalidade da reconstrução de trânsito intestinal colônica em hospital universitário - análise de 42 casos. Rev Bras Coloproct [periódico na Internet]. 2006 Abr-Jun [acesso em 2007 Abr 11]; 26(2):123-7. Disponível em http://www.scielo.br/scielo.php?script=sci_arttex t\&pid=S0101-98802006000200002.

11. Silva AL, Shimizu HE. O significado da mudança no modo de vida da pessoa com estomia intestinal definitiva. Rev Latino-am. Enfermagem [periódico na Internet]. 2006 Jul-Ago [acesso em 2007 Nov 20];
14(4):483-90. Disponível em htpp:/ / www.scielo.br/ scielo.php?script=sci_arttext\&pid=S0104-116920060 00400003\& lang $=p t$

12. Nosella VD, Martins MRI, Netinho JG. Qualidade de vida e atividades cotidianas dos pacientes ostomizados definitivos. SOBEST/ABRASO. Proposta de portaria ministerial - Política Nacional de saúde das pessoas com estomas. Brasília (DF), 2006 [acesso em 2007 Abr 11]. Disponível em www.praticahospitalar. com.br/pratica $\% 2044 /$ pgs/materia

13. Teixeira MG, Almeida M, Teixeira WGJ, Habr-Gama A. Estomias na doença de Crohn. Rev Bras Coloproct [periódico na Internet]. 1999 Abr-Jun [acesso em 2007 Abr 11] 19(2):122-6. Disponível em http:// search.bvsalud.org/regional/?q=Estomias 